\title{
Examination of the vascularization of fetal kidney with three-dimensional power Doppler technique in pregnancies complicated by increased maternal blood pressure
}

\author{
ANDREA SURANYI ${ }^{1, *}$, MIKLOS NOGRADY $^{1}$, ABEL ALTORJAY $^{1}$, \\ TIBOR NYARI ${ }^{2}$, GABOR NEMETH ${ }^{1}$ \\ ${ }^{1}$ Department of Obstetrics and Gynecology, University of Szeged, Szeged, Hungary \\ ${ }^{2}$ Department of Medical Physics and Informatics, University of Szeged, Szeged, Hungary \\ *Corresponding author: Andrea Suranyi; Department of Obstetrics and Gynecology, University of Szeged, 1, Semmelweis, \\ H-6725 Szeged, Hungary; Phone: +36 62 545499; Fax: +36 62 545711; E-mail: gaspar-suranyi.andrea@med.u-szeged.hu
}

(Received: January 8, 2018; Accepted: February 24, 2018)

\begin{abstract}
The goal of this study was to investigate the fetal renal vascularization during the third trimester of gestation and the perinatal outcome in pregnancies diagnosed with hypertension. Depending on the medical history, the cases were divided into two groups: chronic hypertension (CHT) group and gestational hypertension (GHT) group. The vascularization and the volume of kidneys were observed in prenatal period by threedimensional ultrasound. We monitored gestations and perinatal complications. Renal volume and vascularization were detected in 45 cases complicated by GHT and 21 cases with CHT during the 20 -month study period. The alteration in fetal renal volume and vascularization may be an in utero cause of subsequent intrauterine and neonatal complications, such as cesarean section because of fetal distress (36\%), perinatal infection (24\%), treatment in neonatal intensive care unit $(39 \%)$, or increased perinatal mortality (1\%) in affected cases. The results demonstrate that fetuses with depressed vascularization of medullae had 1.5 times the risk of an abnormal outcome compared with the control group. The volume of kidneys had a strong correlation with their vascularization. Detailed ultrasound examinations of renal parenchyma appear to be useful for the prenatal diagnosis of intrauterine hypoxia, allowing the detection of potential pathological fetal conditions in utero.
\end{abstract}

Keywords: fetus, kidney, power Doppler technique, prenatal diagnosis, renal function, three-dimensional ultrasound

\section{Introduction}

Several ultrasound parameters have been proposed as predictors of long-term renal function, such as amniotic fluid index and evaluation of renal parenchyma. However, studies assessing these parameters have not sufficiently yielded high sensitivities and specificities to ensure accurate counseling using ultrasound alone [1-4].

Three-dimensional (3D) quantification of blood flow with the help of power Doppler and virtual organ computer-aided analysis (VOCAL) program provides an estimation of three-dimensional power Doppler (3-DPD) vascular indices: vascularization index (VI), flow index (FI), and vascularization flow index (VFI) [5]. 3-DPD indices reflect not only the blood flow but also the vascularization of the organ as well. A recent study revealed [6] a linear correlation between vascular indices and real blood flow in placental cotyledons in an animal model. Furthermore, these indices have also been studied in some fetal organs and particularly in placenta to predict postnatal prognosis in fetuses displaying intrauterine growth restriction [7].

The screening of the urogenital tract is a very important investigation during pregnancy. The pathological kidney parameters refer to pathological renal development and function. As it is widely known that fetuses with renal failure have poor perinatal outcome, this study aimed at evaluation of renal vascularization using renal

This is an open-access article distributed under the terms of the Creative Commons Attribution-NonCommercial 4.0 International License, which permits unrestricted use, distribution, and reproduction in any medium for non-commercial purposes, provided the original author and source are credited, a link to the CC License is provided, and changes - if any - are indicated. 
3-DPD technique to verify possible correlation of vascular indices to postnatal prognosis.

Hypertensive disorders of pregnancy (HDP) are a major cause of lethal complications in pregnant women [8]. Hypertensive disorders are present in approximately $5 \%-10 \%$ of pregnancies and preeclampsia (PE) complicating about 3\% of pregnancies [9]. A World Health Organization review identified hypertension as the single leading cause of maternal mortality in industrialized countries, accounting for $16 \%$ of maternal deaths [10]. HDP also causes increased fetal and neonatal death $[9,11-13]$. Since 2014, there is a new classification for HDP, which includes chronic hypertension (CHT), gestational hypertension (GHT), PE, de novo or superimposed on CHT, and white coat hypertension [14].

GHT is represented by the disease in placenta with deteriorated function. The CHT is classified as primary or secondary high blood pressure. More than $90 \%$ of cases of CHT belong to primary hypertension, where underlying primary disease is not found in the background. The remaining $10 \%$ pertain to secondary hypertension caused by the illness of other organ systems (kidneys, heart, arteries, or endocrine system).

HDP might decrease the exchange of oxygen between the pregnant woman and fetus and results in chronic hypoxia. Acute and chronic hypoxia also induces circulatory centralization and priority of blood flow to vital organs, such as brain, heart, and adrenal glands [15-17]. During this compensatory mechanism, spasms evolve in the blood vessels of the skin, muscles, kidneys, and the abdominal cavity. The ultrasound examination of the fetal kidneys is a non-invasive procedure and is easy to carry out. We hypothesized that these vascular changes in the kidneys might be a good indicator for fetal well-being in HDP. Although HDP very frequently occurs nowadays, limited data are available about the vascularization and volume changes of fetal kidney in maternal hypertension. Our first objective was to survey the vascularization and the volume of fetal kidney in the third trimester of gestation as well as the perinatal outcome in CHT and GHT.

\section{Materials and Methods}

A prospective case-control study was conducted at the Department of Obstetrics and Gynecology of the University of Szeged (Szeged, Hungary) during a 20-month-long period between 2013 and 2015. The study group consisted of 107 patients: 41 patients with normal parameters (Q), 45 patients with GHT, and 21 patients with CHT.

Inclusion criteria comprised singleton pregnancy complicated by GHT or CHT, maternal disease based on ISSHP [14], and gestational age between 20 and 40 weeks. The GHT and PE are characterized by the new onset of hypertension. The chronic CHT refers to high blood pressure preceding the pregnancy [14]. Controls were fetuses with normal evaluation of morphology at the second trimester scan followed at our department. The patient's withdrawal from the study and/or unavailability for follow-up belonged to exclusion criteria.

Each fetus underwent 3D evaluation of both kidneys to study renal vascularization and volume using a Voluson 730-expert ultrasound machine with 4-8 MHz multifrequency transducer (Kretztechnik, Zipf, Austria). For all cases, power Doppler settings were preestablished before volumetric acquisition of the image: mode cent; smooth: 4/5; FRQ: low; quality: 16; density: 6; enhance: 16; balance: GO150; filter: 2; actual power: $2 \mathrm{~dB}$; pulse repetition frequency: 0.9 .

3D acquisition was performed for each kidney separately. Kidney imaging was set up longitudinally with the point of entry of renal artery visualized by power Doppler. Fetal renal volumes were separately measured for each fetal kidney using the VOCAL program pertaining to the computer software 4D view (GE Medical Systems, Austria; version 10.4). Subsequently, 3-DPD (Fig. 1) was introduced to calculate the vascular indices: VI, FI, and VFI (Fig. 2). The VI, which corresponds to the color voxel/total voxel ratio, measures the quantity of color voxels in the volume of interest, refers to the blood vessels within the volume under examination, and appears as a percentage (vascularity). The FI is the mean color value of all color voxels and represents the average blood flow intensity from 0 to 100 (blood flow). The VFI demonstrating the weighted color voxel/total voxel ratio integrates the data on vessel presence (vascularity) and the number of blood cells transferred (blood flow). The value range extends from 0 to 100 as well.

All cases were followed up in the prenatal period by the staff of our department and in the postnatal period by neonatologists. Two acquisitions per patient were performed by the same operator.

All procedures performed in studies involving human participants were in accordance with the ethical standards of the institutional research committee (ethical approval no.: 32/2014) and with the 1964 Helsinki declaration and its later amendments or comparable ethical standards. Informed consent was obtained from all individual participants included in the study.

\section{Statistical Analysis}

Statistical analyses of the differences between normal and HDP groups were accomplished. The average and standard deviation $( \pm S D)$ for FI, VI, and VFI were compared between normal (Q) and GHT and CHT groups.

We also calculated the mean and standard deviation of the following values: gestational age at birth, the birthweight, the birthlength, and APGAR scores in the 1st, 5 th and 10th minutes. Logistic regression analysis was 


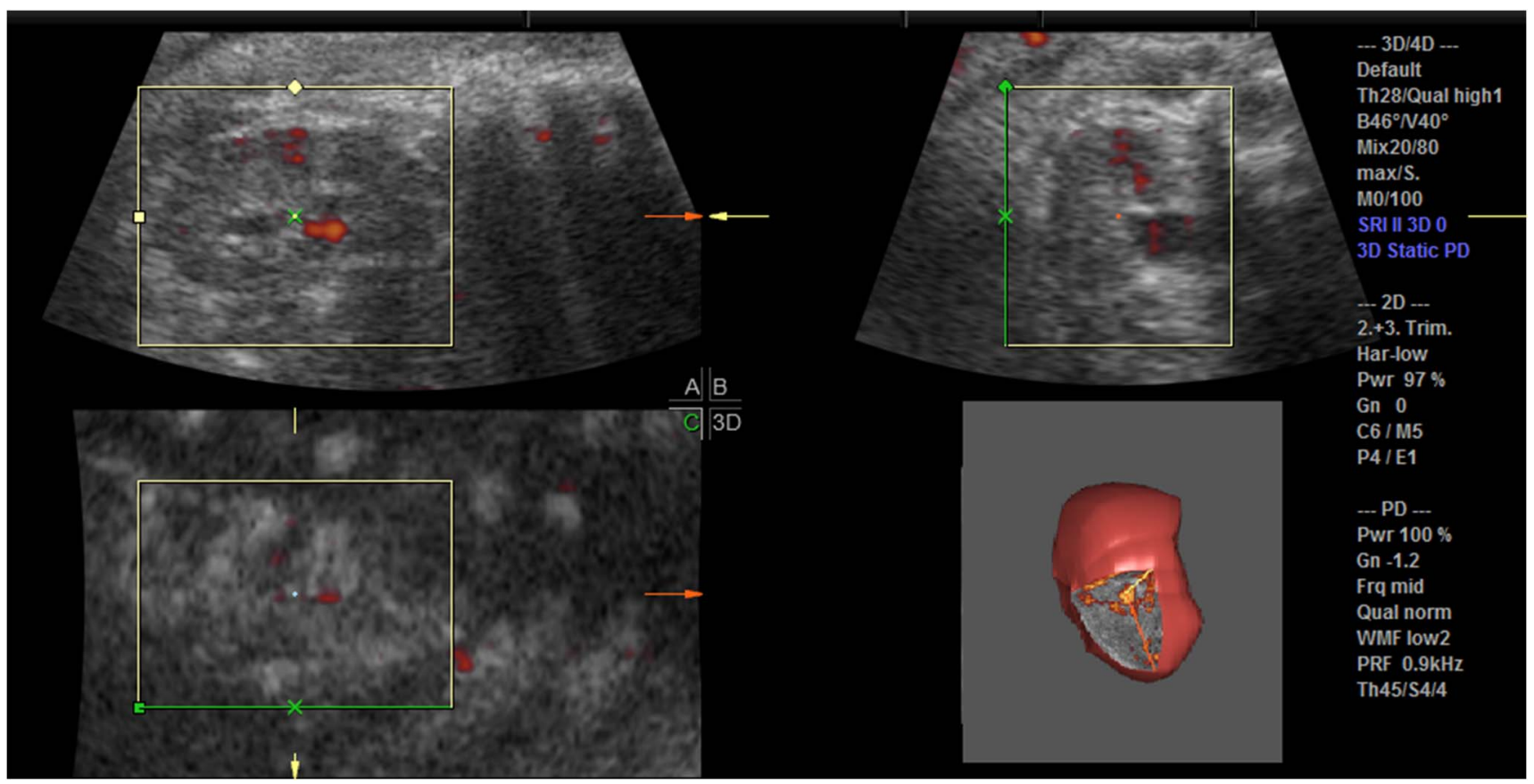

Fig. 1. The fetal kidney image with three-dimensional power Doppler technique

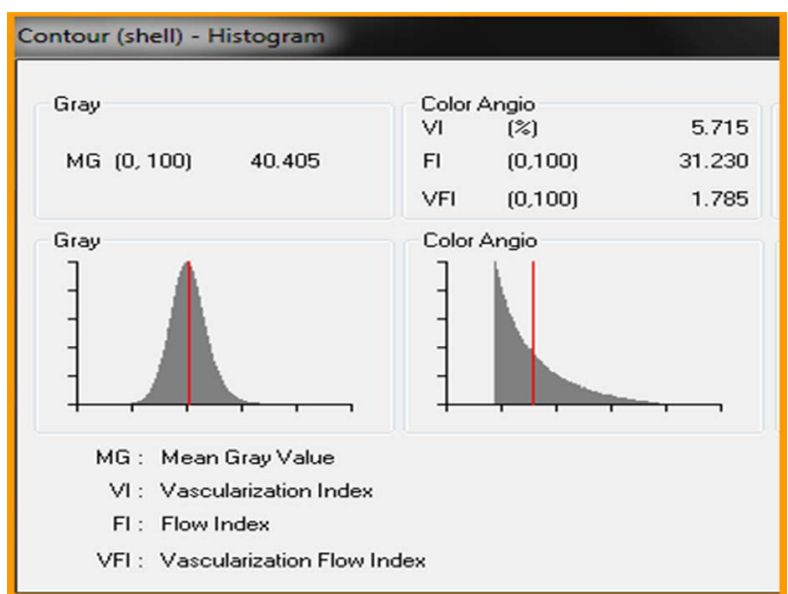

Fig. 2. Sequential quantification of the fetal renal vasculatization indices (VI, FI, and VFI) prepared by the VOCAL software

used to identify the correlation between 3 -DPD indices and the fetal outcome.

The $p$ values less than 0.05 were considered statistically significant.

\section{Results}

The vascular indices of the fetal kidney (VI, FI, and VFI) were significantly decreased (Fig. 3) in the GHT group compared with the control and CHT group (VI: GHT vs. $\mathrm{Q} p=0.000$, GHT vs. CHT $p=0.001$; FI: GHT vs. Q $p=0.000, \mathrm{GHT}$ vs. CHT $p=0.033$; VFI: GHT vs. Q $p=0.000$, GHT vs. CHT $p=0.021)$. There were no significant differences in the volume of the kidneys between the three groups.

Differences between the APGAR scores (Table I): the 1-min APGAR scores were significantly decreased in the GHT group compared with the $\mathrm{Q}$ group $(p=0.025)$. In the CHT group, the $5-(p=0.042)$ and $10-\mathrm{min}(p=$ $0.032)$ APGAR scores were significantly decreased compared with the $\mathrm{Q}$ group. Between the rest of the values, no significant difference could be found. After the birth, we measured the $\mathrm{pH}$ level from the umbilical vein, the birth weight, and the birth length. There were no significant differences between the three groups (Table I).

We observed the newborns in neonatal intensive care unit (NICU), if there were respiratory arrest, polycythemia, hypoglycemia, or need for therapy (Table II). The newborns needed transportation to the NICU in the CHT group: $21.7 \%$ out of group; GHT group: $17.2 \%$ out of group; and control group: $2.4 \%$ out of group. It made a significant difference $(p=0.021)$ among the groups.

\section{Discussion}

This is the first study in which the vascularization of the fetal kidney was investigated in HDP. The monitoring of depression of renal vascularization can be quantified and it is significantly lower in fetuses with GHT that may evolve renal circulatory insufficiency, and may provide us with valuable new information helping the maternal care. Contrary to the GHT, the renal vascularization was not reduced 

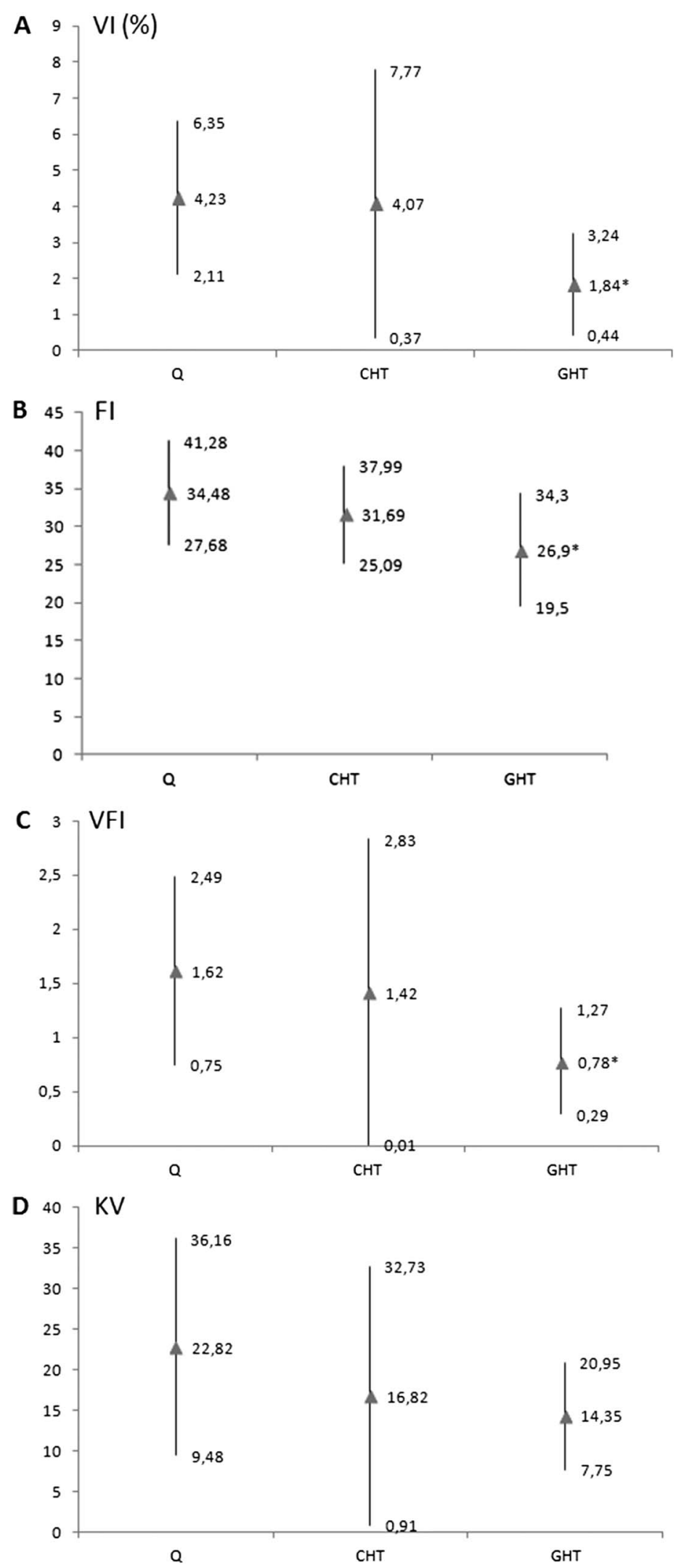

Fig. 3. Vascular indices and volume of the fetal kidney in the control (Q) group $(n=41)$, chronic hypertension (CHT) group ( $n=21)$, and gestational hypertension (GHT) group $(n=45)$. The solid triangles imply mean values and the two ends of the vertical lines represent the $( \pm)$ standard deviations. The symbol * indicates significant differences compared with the Q group. (A) VI: vascularization index, (B) FI: flow index, (C) VFI: vascularization flow index, (D) KV: kidney volume. * $p<0.05$ 
Table I Parametric values about the APGAR scores (Apg), umbilical pH (umb pH), birth weight (BW), and birth length $(\mathrm{BL})$ of newborns

\begin{tabular}{lccc} 
& Q & CHT & GHT \\
Apg 1 & $9.41(0.87)$ & $8.82(1.7)$ & $8.71(1.4)^{*}$ \\
Apg 5 & $9.9(0.3)$ & $9.44(1.31)^{*}$ & $9.67(0.62)$ \\
Apg 10 & $10(0.0)$ & $9.7(0.77)^{*}$ & $9.88(0.45)$ \\
Umb pH & $7.26(0.09)$ & $7.24(0.1)$ & $7.25(0.07)$ \\
BW (g) & $3287(434)$ & $3070(711)$ & $3153(705)$ \\
BL $(\mathrm{cm})$ & $48.88(2.63)$ & $48.86(2.44)$ & $48.5(3.45)$ \\
\hline
\end{tabular}

Standard deviation values are indicated in the brackets. The symbol * indicates significant difference compared with the $\mathrm{Q}$ group. Q: control; CHT: chronic hypertension; GHT: gestational hypertension. ${ }^{*} p<0.05$

Table II Non-parametric values concerning the percentage of newborns with respiratory arrest, polycythemia, hypoglycemia, or necessity of treatment at NICU

$\begin{array}{lccc} & \mathrm{Q}(n=41)(\%) & \mathrm{CHT}(n=23)(\%) & \mathrm{GHT}(n=64)(\%) \\ \text { Respiratory arrest } & 2.44 & 13.04 & 14.06 \\ \text { Polycythemia } & 2.44 & 4.38 & 5.36 \\ \text { Hypoglycemia } & 2.44 & 0.00 & 4.69 \\ \text { Transportation to NICU } & 2.44 & 21.74 & 17.19^{*}\end{array}$

Q: control; CHT: chronic hypertension; GHT: gestational hypertension; NICU: neonatal intensive care unit. ${ }^{*} p<0.05$

in CHT. CHT does not cause reduction of the vascularization in the placenta. In these cases, the human body can compensate the consequences of the hypertension.

The number of the newborns was significantly higher in the GHT group requiring neonatal intensive care. This also points to the fact how important it would be to identify patients as soon as possible with GHT, and check up this high-risk group.

The power Doppler shows a high sensitivity to depict a vascular tree, because it is based on Doppler signal amplitude rather than average frequency values, unlike the color Doppler. Moreover, it does not generate the effects of aliasing and color maps are independent of the angle of insonation. The 3-DPD not only allows a full and adequate study of the vascular placental tree by identifying the different branches of villi $[18,19]$ but also produces a quantitative assessment of the number of vessels through the vasculature and blood flow index, by applying flow rates and vascularization. By the $3 \mathrm{D}$ method, vascular villi of the first, second, and the third order can be seen and thus a higher percentage is achieved in comparison with 2D Doppler technique.

Low VI values are interpreted as a decrease in the number of vessels within the placenta. The decreased FI values can be viewed as a reduction in placental blood flow due to increasing placental resistance. Low VFI values manifest a decrease in the number of vessels in the placenta, as well as a reduction of blood flow. If reduced, the three indices reveal that there is a decrease in speed, intensity, and placental blood perfusion.
Fetal-downregulated vascularization had influence on fetal renal development $[18,20]$. This is an important conclusion, because it suggests that early identification of women at low risk of HPD can be improved to obtain prophylactic treatment and intense surveillance can be initiated at appropriate time.

Funding sources: This study was supported by the Hungarian EFOP3.6.1-16-2016-00008 grant.

Authors' contribution: AS carried out the majority of the literature research, prepared the manuscript, prepared the study design, and analyzed the $3 \mathrm{D}$ images. $\mathrm{MN}$ and $\mathrm{AA}$ performed obstetrical $2 \mathrm{D}$ and 3D ultrasound, compiled data, did some literature research, and supervised the manuscript. TNy carried out the statistical analyses and supervised the manuscript. GN supervised the study design and manuscript.

Conflict of interest: The authors declare no conflict of interest.

Acknowledgements: The authors would like to thank Cedars Sinai Medical Center's International Research and Innovation in Medicine Program, the Association for Regional Cooperation in the Fields of Health, Science and Technology (RECOOP HST Association) for their support of our organization as participating Cedars - Sinai Medical Center - RECOOP Research Centers (CRRC).

\section{Abbreviations}

3D : three-dimensional

3-DPD : three-dimensional power Doppler 


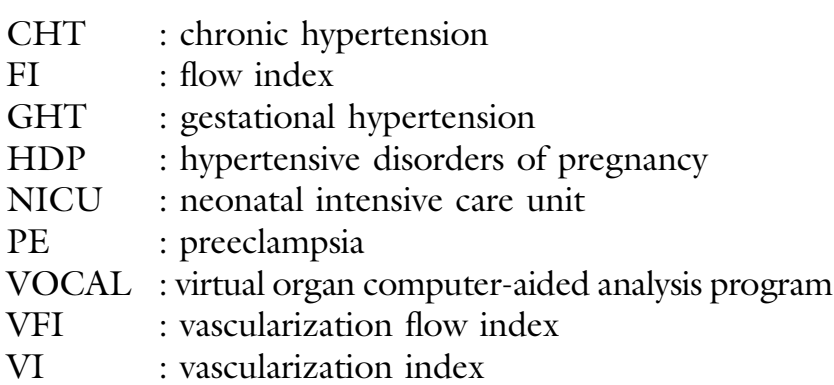

\section{References}

1. Qureshi F, Jacques SM, Seifman B, Quintero R, Evans MI, Smith C, Johnson MP: In utero fetal urine analysis and renal histology correlate with the outcome in fetal obstructive uropathies. Fetal Diagn Ther 11, 306-312 (1996)

2. Mussap M, Fanos V, Pizzini C, Marcolongo A, Chiaffoni G, Plebani M: Predictive value of amniotic fluid cystatin C levels for the early identification of fetuses with obstructive uropathies. BJOG 109, 778-783 (2002)

3. Surányi A, Pál A, Streitman K, Pintér S, Kovács L: Fetal renal hyperechogenicity in pathological pregnancies. J Perinat Med 25, 274-279 (1997)

4. Surányi A, Retz C, Rigo J, Schaaps JP, Foidart JM: Fetal renal hyperechogenicity in intrauterine growth retardation: Importance and outcome. Pediatr Nephrol 16, 575-580 (2001)

5. Pairleitner H, Steiner H, Hasenoehrl G, Staudach A: Threedimensional power Doppler sonography: Imaging and quantifying blood flow and vascularization. Ultrasound Obstet Gynecol 14, 139-143 (1999)

6. Morel O, Pachy F, Chavatte-Palmer P, Bonneau M, Gayat E, Laigre P, Evain-Brion D, Tsatsaris V: Correlation between uteroplacental three-dimensional power Doppler indices and true uterine blood flow: Evaluation in a pregnant sheep model. Ultrasound Obstet Gynecol 36, 635-640 (2010)

7. Molnár A, Surányi A, Nyári T, Németh G, Pál A: Examination of placental three-dimensional power Doppler indices and perinatal outcome in pregnancies complicated by intrauterine growth restriction. Int J Gynaecol Obstet 129, 5-8 (2015)

8. Hwang J-W, Park S-J, Oh S-Y, Chang SA, Lee SC, Park SW, Kim DK: The risk factors that predict chronic hypertension after delivery in women with a history of hypertensive disorders of pregnancy. Medicine (Baltimore) 94, el747 (2015)

9. Hutcheon JA, Lisonkova S, Joseph KS: Epidemiology of preeclampsia and the other hypertensive disorders of pregnancy. Best Pract Res Clin Obstet Gynaecol 25, 391-403 (2011)

10. Khan KS, Wojdyla D, Say L, Gülmezoglu AM, Van Look PF: WHO analysis of causes of maternal death: A systematic review. Lancet 367, 1066-1074 (2006)

11. Jain L: Effect of pregnancy-induced and chronic hypertension on pregnancy outcome. J Perinatol 17, 425-427 (1997)

12. Vanek M, Sheiner E, Levy A, Mazor M: Chronic hypertension and the risk for adverse pregnancy outcome after superimposed preeclampsia. Int J Gynaecol Obstet 86, 7-11 (2004)

13. Zhang J, Cai WW, Lee DJ: Pregnancy-induced hypertension and early neonatal death: A case-control study. Am J Perinatol 10, 401-403 (1993)

14. Tranquilli AL, Dekker G, Magee L, Roberts J, Sibai BM, Steyn W, Zeeman GG, Brown MA: The classification, diagnosis and management of the hypertensive disorders of pregnancy: A revised statement from the ISSHP. Pregnancy Hypertens 4, 97-104 (2014)

15. Cohn HE, Sacks EJ, Heymann MA, Rudolph AM: Cardiovascular responses to hypoxemia and acidemia in fetal lambs. Am J Obstet Gynecol 120, 817-824 (1974)

16. Kjellmer I, Karlsson K, Olsson T, Rosén KG: Cerebral reactions during intrauterine asphyxia in the sheep. I. Circulation and oxygen consumption in the fetal brain. Pediatr Res 8, 50-57 (1974)

17. Kamitomo M, Alonso JG, Okai T, Longo LD, Gilbert RD: Effects of long-term, high-altitude hypoxemia on ovine fetal cardiac output and blood flow distribution. Am J Obstet Gynecol 169, 701-707 (1993)

18. Surányi A, Nyári T, Pál A: What is biparietal diameter/kidney length ratio in cases with renal hyperechogenicity? Pediatr Nephrol $18,14-17$ (2003)

19. Surányi A, Streitman K, Pál A, Nyári T, Retz C, Foidart JM, Schaaps JP, Kovács L: Fetal renal artery flow and renal echogenicity in the chronically hypoxic state. Pediatr Nephrol 14, 393-399 (2000)

20. Suranyi A (2000): Prenatal and postnatal evaluation of foetal renal hyperechogenicity in pregnancies complicated with pre-eclampsia and intrauterine growth retardation. Dissertation, University of Szeged. Retrieved from http://doktori.bibl. u-szeged.hu/2154/ 